\title{
"The Dichotic Digit Test" as an Index Indicator for Hearing Problem in Dementia: Systematic Review and Meta-Analysis
}

\author{
${ }^{1}$ UCL Ear Institute, Faculty of Brain Science, University College \\ London, London, United Kingdom \\ ${ }^{2}$ Department of Otolaryngology, Faculty of Medicine, Chulalongkorn \\ University, Pathumwan, Bangkok, Thailand \\ ${ }^{3}$ Dementia Research Centre, Faculty of Brain Science, University \\ College London, London, United Kingdom \\ ${ }^{4}$ Division of Psychology and Language Science, Faculty of Brain \\ Sciences, University College London, Gower Street, United Kingdom \\ ${ }^{5}$ Division of Psychiatry, Faculty of Brain Sciences, University College \\ London, Gower Street, United Kingdom \\ ${ }^{6}$ NIHR Biomedical Research Centre Hearing and Deafness, London, \\ United Kingdom
}

Nattawan Utoomprurkporn, MD, Msc ${ }^{1,2}$ Chris J.D. Hardy, $\mathrm{PhD}^{3}$ Joshua Stott, DclinPsy, PhD 4 Sergi G. Costafreda, MD, $\mathrm{PhD}^{5}$ Jason Warren, MD, PhD ${ }^{3}$ Doris Eva Bamiou, MD, Msc, PhD ${ }^{1,6}$

Address for correspondence Nattawan Utoomprurkporn, MD, Msc,

J Am Acad Audiol 2020;31:646-655.

\begin{abstract}
Keywords

- dichotic

- dementia

- (central) auditory process

- neurodegeneration

- cognition

Background Patients with dementia commonly have problems processing speech in the presence of competing background speech or noise. This difficulty can be present from the very early stages of dementia, and may be a preclinical feature of Alzheimer's disease.

Purpose This study investigates whether people with dementia perform worse on the dichotic digit test (DDT), an experimental probe of speech processing in the presence of competing speech, and whether test performance may predict dementia onset.

Research Design Systematic review and meta-analysis.

Data Collection and Analysis A literature search was conducted in Medline, Embase, Scopus, and Psycinfo. We included (1) studies that included people with a diagnosis of dementia and a healthy control group with no cognitive impairment; (2) studies that reported results from a DDT in a free-recall response task; and (3) studies that had the dichotic digit mean correct percentage score or right-ear advantage, as outcome measurements.

Results People with dementia had a lower DDT total score, with a pooled mean difference of $18.6 \%$ (95\% confidence interval [CI]: 21.2-15.9). Patients with dementia had an increased right-ear advantage relative to controls with a pooled difference of 24.4\% (95\% Cl: 21.8-27.0).

Conclusion The DDT total scores are lower and the right-ear advantage increased in cognitively impaired versus normal control participants. The findings also suggest that the reduction of dichotic digit total score and increase of right-ear advantage progress as cognitive impairment increases. Whether abnormalities in dichotic digit scores could predict subsequent dementia onset should be examined in further longitudinal studies.
\end{abstract}

received

September 26, 2019

accepted after revision

February 18, 2020

published online

December 9, 2020
(C) 2020. American Academy of

Audiology. All rights reserved.

Thieme Medical Publishers, Inc.,

333 Seventh Avenue, 18th Floor,

New York, NY 10001, USA
Dol https://doi.org/

10.1055/s-0040-1718700. ISSN 1050-0545. 
People with cognitive impairment often have problems perceiving and processing target speech in the presence of background noise or competing speech, which may be understood as a specific form of auditory processing difficulty. This difficulty often presents at very early stages of dementia, ${ }^{1}$ and may even precede the diagnosis of dementia by several years. ${ }^{2}$ This transitional stage from normal cognition to dementia, during which there is some cognitive decline but not severe enough to interfere with the person's performance of activities of daily living significantly, is known as mild cognitive impairment (MCI).,4 People with $\mathrm{MCI}$ may also experience difficulties processing speech in background noise. ${ }^{5}$ Similarly, abnormalities of auditory cortical-evoked sensory potentials predate clinical symptoms in young carriers of pathogenic Alzheimer's disease (AD) mutations. ${ }^{6}$

Dichotic speech tests are one category of tests in the auditory processing test battery that assesses binaural integration and/or binaural separation of competing speech information in the free recall task. The "dichotic digit test" (DDT) in particular has been proposed as a screening test for central auditory processing pathway abnormalities due to its easy application and short administering time, along with its resistance to peripheral hearing loss. ${ }^{7}$ The most commonly used paradigm is the two-digit pair paradigm, where two digits are presented to each ear at the same time ${ }^{8}$ and at the suprathreshold level to ensure that even the patient with hearing loss can hear this. ${ }^{8}$ Several researchers have suggested that this DDT paradigm may be useful in assessing auditory processing in individuals with dementia. ${ }^{9,10}$

Other more cognitively challenging variations of DDTs were also used in previous research, to avoid the ceiling effects found in two digit pairs test paradigms, ${ }^{11}$ such as using three-digit pairs ${ }^{12}$ or randomly presented one-, two-, and three-pair dichotic digit stimuli. ${ }^{13}$ However, the twodigit paradigm was more accurate in discriminating between control and $\mathrm{AD}$ groups due to higher error rates even in controls with three digits. ${ }^{5}$

The difference between the correct response percentage score of the right and the left ear is called "the right-ear advantage." A right-ear advantage is observed when participants have a better recall of stimuli presented to the right than the left ear, as first described by Kimura in $1961 .^{14}$ This is because, for the majority of people, the left hemisphere is regarded as the language-dominant hemisphere with some variation. ${ }^{15}$ When the target speech signal is presented to the right ear, it can be transmitted directly via the cross-pathway to be processed in the left hemisphere. However, when the target is coming from the left ear, it is first relayed to the right hemisphere, and then via the corpus callosum to be processed in the primary auditory cortex on the left. The normative data in general population showed an increased right ear advantage for the younger (age 6-12 years) and the older (over 60 years) cohorts, which may indicate underlying early development maturation and age-related degenerative changes of the pathway. ${ }^{16}$ Consistent with this functional neuroanatomy, patients with corpus callosum white matter lesions show an increased difference in the performance score of the two ears, with the expected right-ear advantage. ${ }^{17,18}$ However, other structural and neural plasticity processes beyond the corpus callosum can also play a role in dichotic listening performance. In children with corpus callosum agenesis, while the right ear advantage is significantly different to that of age-matched controls in early stages of development, but this difference is not as marked when they get older. ${ }^{19,20}$

Interestingly, in addition to several brain-structure changes observed early in the course of $A D$, such as in the hippocampi and precuneus, ${ }^{21}$ alterations in the corpus callosum have also been observed. ${ }^{22}$ Myelin sheath breakdown of regions such as the corpus callosum, which myelinate later during development, may be more rapid among older adults who are at risk of developing $\mathrm{AD}^{23}$ Parsimoniously, the poorer performance in DDT in the left ear in AD subjects compared with controls may index corpus callosum changes. DDT may, therefore, represent a sensitive probe of central auditory dysfunction in the context of neurodegenerative diseases. In addition, other dementia biomarkers such as cerebrospinal fluid total-tau and P-tau levels also show an association with right-ear advantage in older adults with a family history positive for AD. ${ }^{24}$

This systematic review and meta-analysis examines the evidence for associations between right-ear advantage score and total score on the free recall/divided attention task in the DDT with all-cause dementia but with a specific focus on AD. The potential of using DDT as a predictor of dementia is also discussed.

Specific aims of this systematic review are to investigate whether:

- Adults with dementia perform worse on DDT and have wider right ear advantage scores than healthy control participants.

- Abnormal DDT findings could be used to predict future dementia onset.

\section{Method}

This systematic review and meta-analysis follows the Cochrane guidance for systematic reviews and the Preferred Reporting Items for Systematic Reviews and Meta-Analyses (PRISMA) guidance. The full protocol is published via Protocol registration CRD42018100391 on the PROSPERO register database (International Prospective Register of Systematic Reviews).

The literature search was conducted on May 27, 2018 in Medline (via Pubmed), Embase, Scopus, and Psycinfo, to ensure coverage of all published materials in medicine, psychology, and other fields. We included studies with the following criteria for our review: (1) studies that included people with a diagnosis of dementia and a healthy control group with no cognitive impairment; (2) studies that reported results from a DDT in a free-recall response task; and (3) studies that had the DDT mean correct percentage score and standard deviation (SD) or median and interquartile range or right-ear advantage, defined as the difference of the DDT score between right and left ears, as outcome measurements. The search keywords included dementia, cognitive dysfunction, and Alzheimer and dichotic digit (see - Appendix 1 for details). All papers 
meeting the above criteria, with retrievable full texts written in English, found in the above-mentioned databases on the search date were included. All study designs were included.

\section{Study Selection}

The studies were selected by two reviewers (N.U. and D.E.B.) after reviewing information for the study's inclusion criteria from the titles and abstracts. When in doubt, the study full text was also reviewed as part of the study selection process and discussed. When there was no consensus between the two reviewers, the studies were discussed with a third reviewer (S.G.C.) to seek a final conclusion among the reviewers. All studies which met the eligibility criteria were included in the systematic review.

\section{Data Collection Process}

Data extracted from each paper included the participants' dichotic digit scores, average ages, dementia diagnostic procedure, dementia type, and recruitment sites. These were collated in a Microsoft Excel spreadsheet. Risk of bias was evaluated for each study using the NIH-Quality Assessment Tool for Observational Cohort and Cross-Sectional Studies ${ }^{25}$ by N.U. and D.E.B. Publication bias was evaluated with a funnel plot. Asymmetry of the funnel plot which plotted the effect estimate (mean difference, MD) against the standard error of the MD (SE [MD]) of the included studies may indicate potential publication bias. ${ }^{26,27}$

The paper data were analyzed with Review Manager Version $5.3,{ }^{28}$ to create meta-analytic summary estimates of the pooled data for the total DDT mean score (combined right and left ears) and ear-specific DDT mean score (to calculate the right-ear advantage by right-ear pooled mean score and left-ear pooled mean score difference). These scores were compared across dementia versus noncognitively impaired control participants using a random effects model (inverse variance method). ${ }^{29}$ The consistency of the data in the meta-analysis was evaluated with chi-square $\left(\mathrm{X}^{2}\right)$ and $I$-square $\left(I^{2}\right)$ heterogeneity tests.

Separate meta-analyses were performed for all included papers. Papers with cross-sectional designs were used to study the association between DDT scores and dementia. Papers with longitudinal designs were used to investigate the use of DDT score as a predictor for future dementia onset.

\section{Results}

\section{Study Selection and Characteristics}

From the database search, we retrieved 34 papers from Pubmed, 41 papers from Scopus, 29 papers from Embase, and 14 papers from Psycinfo. One additional paper was found from the reference lists. The PRISMA (Preferred Reporting Items for Systematic Reviews and Meta-Analyses) flow diagram of this systematic review is shown in - Fig. 1.

A total of eight papers (two longitudinal and six crosssectional) were included in the systematic review. The diagnostic criteria for dementia, type of dementia, and $\mathrm{MCI}$ for each paper are listed in - Supplementary Table 1 (online only).
Baseline characteristics of the two samples (dementia group and noncognitively impaired group) were analyzed. Age and hearing level did not differ significantly between the groups in any of the cohorts, except in one retrospective cross-sectional study, in which the dementia group was slightly older and had more hearing loss than the healthy control group. ${ }^{9}$ In the metaanalysis, there was no significant difference in pooled mean age between the two groups, with a mean age difference of 1.52 years (95\% confidence interval [CI]: -1.34 to 4.38 ).

There was no significant difference between the pooled mean level of hearing loss between the two groups with a mean pure-tone average difference of $2.93 \mathrm{~dB}(95 \% \mathrm{CI}$ : -2.46 to 8.33). There was no significant difference in sex distribution across people with dementia and healthy controls $(p=0.12)$. Years of education were reported only in two papers. ${ }^{9,12}$ There was a slight pooled MD of 1.27 years, with healthy controls having more education than people with dementia (95\% CI: 0.06 to -2.48 ).

\section{Risk of Bias}

Six of the eight studies included and summarized in -Supplementary Table 1 (online only) provided details about the recruitment process and diagnostic criteria for each group; two studies did not report this information. ${ }^{30,31}$

None of the studies provided a sample size calculation, meaning that they may have been underpowered. None of the studies gave information on methods of assessor blinding while testing the participants, meaning that there was a potential observer bias in all of the studies reported.

\section{Qualitative Synthesis}

\section{Cross-Sectional Studies}

All six papers showed consistent results: decreased total dichotic digit score and/or increased right-ear advantage in dementia subjects compared with controls. ${ }^{5,9,10,12,30,32}$ Two studies reported total DDT scores but not separate right- and left-ear scores. ${ }^{9,32}$

After full-text review and extraction of the data, only four of the six papers were included in the data extraction for meta-analysis. Of the two papers that were not included, one presented data only in a graph format without any variance data. $^{30}$ Two papers presented data from the same cohort, ${ }^{9,32}$ so only one dataset was used for the meta-analysis.

The baseline data from the two longitudinal studies could not be included in the cross-sectional meta-analysis for separate reasons. Gates et $\mathrm{al}^{2}$ did not include a dementia group at baseline since they excluded the prevalent cases of dementia from their study: the main purpose of this research was to monitor the incidence of future dementia diagnosis and whether the DDT could be used to predict future dementia in a cohort of people without dementia at baseline. Idrizbegovic et $\mathrm{al}^{31}$ had presented their baseline data in a crosssectional paper published by the same researchers in 2011.

\section{Longitudinal Studies}

It was not possible to combine the data from the two longitudinal studies ${ }^{2,31}$ for the purposes of a meta-analysis as the 


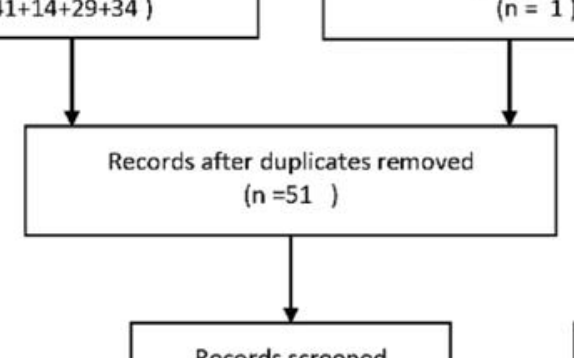

Additional records identified through other sources $(n=1)$
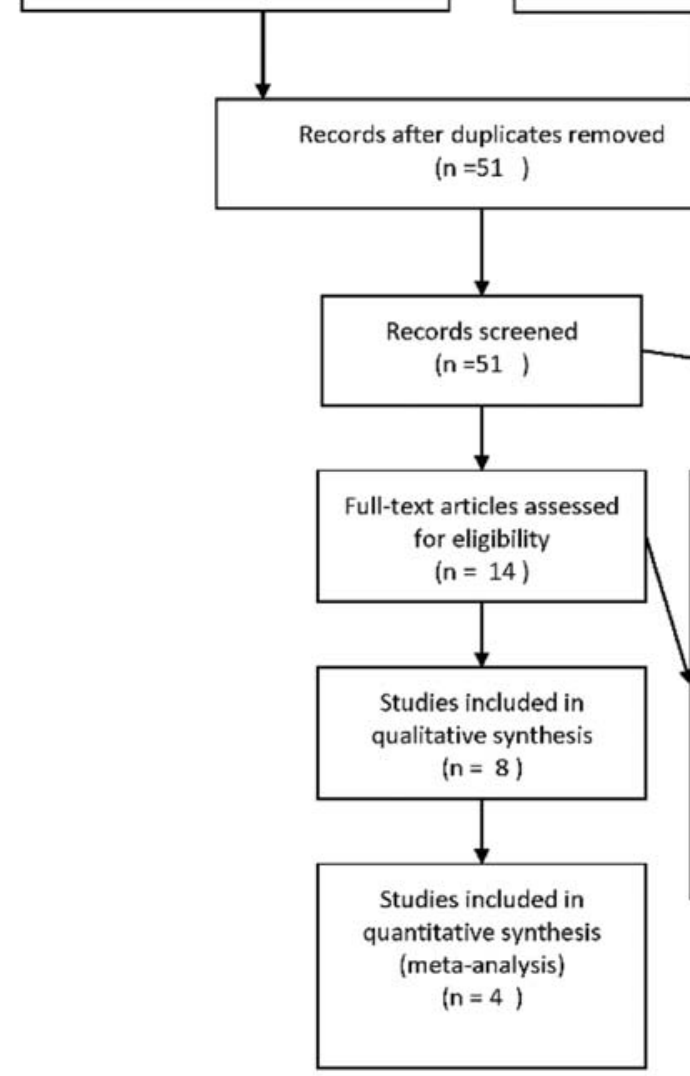

Records excluded ( $\mathrm{n}=37$ )

From: Moher D, Liberati A, Tetzlaff J, Altman DG. The PRISMA Group (2009). Preferred Reporting Items for Systematic Reviews and Meta. Analyses: The PRISMA Statement. PLOS Med 6(7): e1000097. doi:10.1371/journal.pmed1000097

For more information, visit www.prisma-statement.ore.

Fig. 1 PRISMA flow diagram.

article written by Idrizbegovic et $\mathrm{al}^{31}$ was a preliminary report that contained limited details and did not report variance data. This prospective study had a short follow-up time of 1.5 years. ${ }^{31}$ At baseline, there was no significant hearing loss at any frequency between 0.125 and $2 \mathrm{kHz}$ in any ear and no significant between-group differences in hearing threshold levels at any frequencies, and in either ear, with no significant interaural differences. The average left-ear DDT score was lower in people with dementia (mean $=60 \%$ ) than in people with subjective memory complaint (mean $=90 \%$ ). After 1.5 years, the score in the dementia group significantly decreased from baseline, and this difference was significantly different from controls. ${ }^{31}$ Since the data for the right-ear DDTscore were not reported at follow-up, it was not possible to calculate a total mean score. However, the paper reported no significant difference in the right-ear dichotic digit score from baseline scores in all three groups (subjective memory complaint, $\mathrm{MCI}$, and dementia).

The other longitudinal study ${ }^{2}$ looked at DDT scores from a population-based longitudinal study of aging and dementia with a follow-up from 10 to 48 months after the initial hearing tests. The baseline mean DDT score for participants who later developed dementia was $58 \%(\mathrm{SD}=18)$, which was significantly worse than the $75 \%(S D=16)$ seen in the group of participants who did not develop dementia. Moreover, when using an $80 \%$ DDT score as a cut point, participants who failed the test at baseline were more likely to develop dementia in future, with a hazard ratio of $7.0(95 \% \mathrm{CI}:-1.6 \text { to } 31.0)^{2}$ 


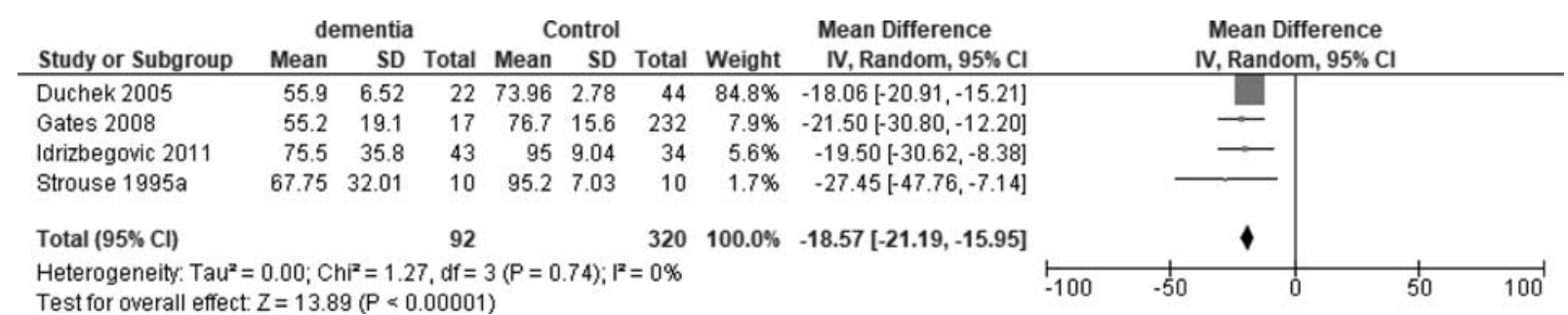

Fig. 2 Total dichotic digits score of dementia versus noncognitively impaired controls. For the mean difference approach, the standard deviations and the sample sizes are used together to calculate the weight given to each study. The square represents the weighted mean difference while the diamond represents the pooled mean difference. Cl, confidence interval; IV, inverse variance; SD, standard deviation.

\section{Meta-Analytic Synthesis of Results (Cross-Sectional Studies)}

\section{Total Dichotic Digit Mean Scores}

Dementia versus Noncognitively Impaired Controls

Four papers were included in this quantitative analysis. $5,9,10,12$ The mean pooled data of the total DDT score were significantly lower in the dementia group compared with noncognitively impaired controls, with a MD of $-18.57 \%$ (95\% CI: -21.19 to -15.95$)$ as shown in - Fig. 2. Heterogeneity tests showed absence of heterogeneity across all the included studies $\left(X^{2}=1.27, \mathrm{df}=3, I^{2}=0 \%\right.$ ) and there was no asymmetry in the funnel plot (see - Supplementary Fig. 1, online only), indicating no publication bias.

\section{Dementia versus Mild Cognitive Impairment}

Three papers were included in this quantitative analysis., ${ }^{5,12}$ The mean pooled data for the dementia group were significantly lower than those seen in the MCI group, with a MD of $-13.84 \%$ (95\% CI: -20.09 to -7.59$)$ as shown in - Fig. 3. Heterogeneity tests showed moderate heterogeneity across all the included studies $\left(X^{2}=3.86, \mathrm{df}=2\right.$, $I^{2}=48 \%$ ).

Due to the heterogeneity of the data included in the comparison between people with dementia and $\mathrm{MCI}$, a sensitivity analysis was performed by excluding the data presented by Gates et al, ${ }^{9}$ as the diagnostic criteria for $\mathrm{MCI}$ were different to the criteria used by the other studies. This analysis did not substantially change the results, as the pooled dementia group had a significantly lower mean score than the MCI group, with a MD of $-16.62 \%(95 \% \mathrm{CI}:-19.60$ to -13.63 ) as shown in - Supplementary Table 2 (online only).
Heterogeneity tests showed homogeneity among all the included studies ( $X^{2}=0.08, \mathrm{df}=1, I^{2}=0 \%$ ).

Mild Cognitive Impairment versus Noncognitively Impaired Controls

Three papers ${ }^{5,9,12}$ were included in this quantitative analysis. ${ }^{5,9,12}$ The mean pooled data of the total dichotic digit score for the pooled MCI group and the pooled noncognitively impaired control group were not significantly different, with a MD of $-6.89 \%$ (95\% CI: -15.54 to 1.76 ) as shown in - Fig. 4 . Heterogeneity tests showed high heterogeneity among all the included studies $\left(X^{2}=29.55, \mathrm{df}=2, I^{2}=93 \%\right.$ ).

A sensitivity analysis was again performed by excluding the Gates et $\mathrm{al}^{9}$ data. This did not substantially change the results, as the $\mathrm{MCl}$ group total dichotic digit score was not significantly different from noncognitively impaired controls, with a MD score of $-1.79 \%$ (95\% CI: -3.99 to 0.40 ) as shown in - Supplementary Table 3 (online only). Subsequent heterogeneity tests showed homogeneity among all the included studies $\left(X^{2}=1.17, \mathrm{df}=1, I^{2}=15 \%\right)$.

\section{Difference in Ear-Specific Dichotic Digit Score (Right-Ear Advantage)}

\section{Dementia}

Three papers were included in this quantitative analysis. ${ }^{5,10,12}$ The difference between the mean pooled DDT scores in each ear for the dementia group was statistically significant. The right-ear mean dichotic digit score average was higher than the left-ear mean score by $24.38 \%$ (95\% CI: 21.76-26.99), as shown in -Fig. 5. Heterogeneity tests showed homogeneity among all the included studies $\left(X^{2}=1.32, \mathrm{df}=2, I^{2}=0 \%\right)$.

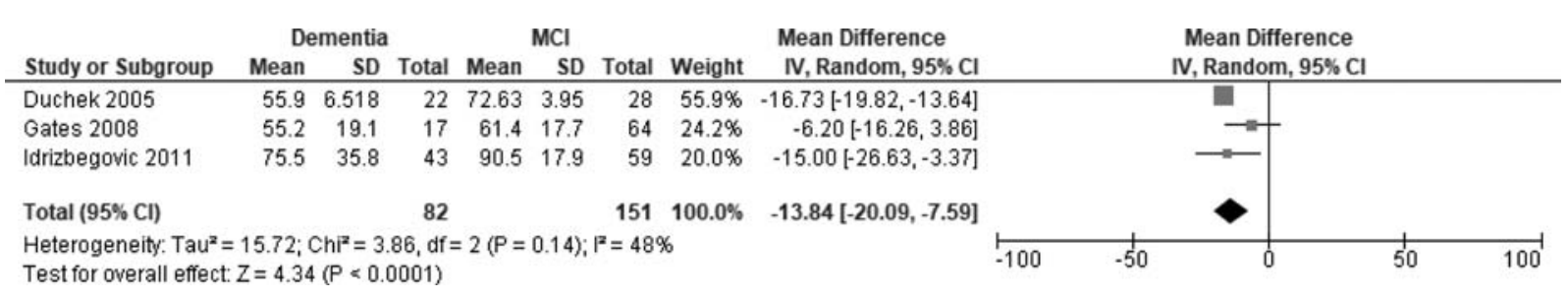

Fig. 3 Total dichotic digits score of dementia versus mild cognitively impaired. For the mean difference approach, the standard deviations and the sample sizes are used together to calculate the weight given to each study. The square represents the weighted mean difference while the diamond represents the pooled mean difference. $\mathrm{Cl}$, confidence interval; IV, inverse variance; $\mathrm{MCl}$, mild cognitive impairment; SD, standard deviation. 


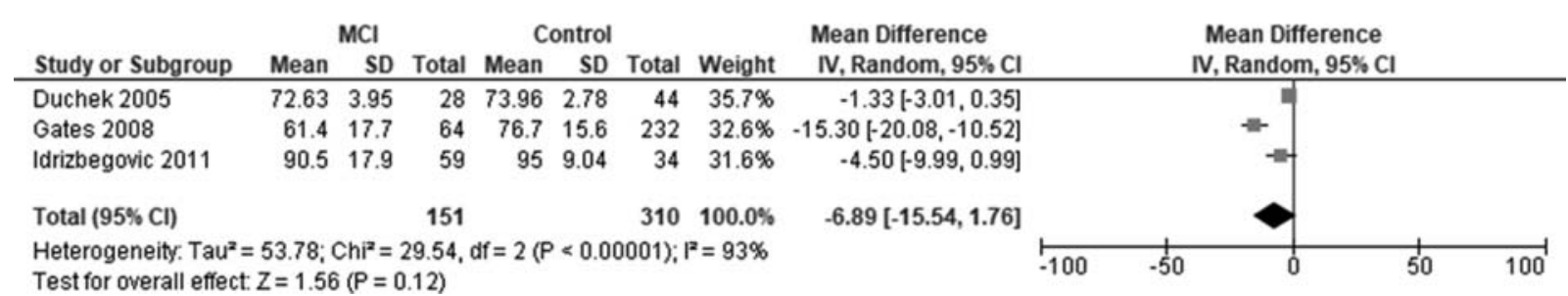

Fig. 4 Total dichotic digits score of mild cognitive impaired versus noncognitively impaired controls. For the mean difference approach, the standard deviations and the sample sizes are used together to calculate the weight given to each study. The square represents the weighted mean difference while the diamond represents the pooled mean difference. $\mathrm{CI}$, confidence interval; IV, inverse variance; $\mathrm{MCI}$, mild cognitive impairment; SD, standard deviation.

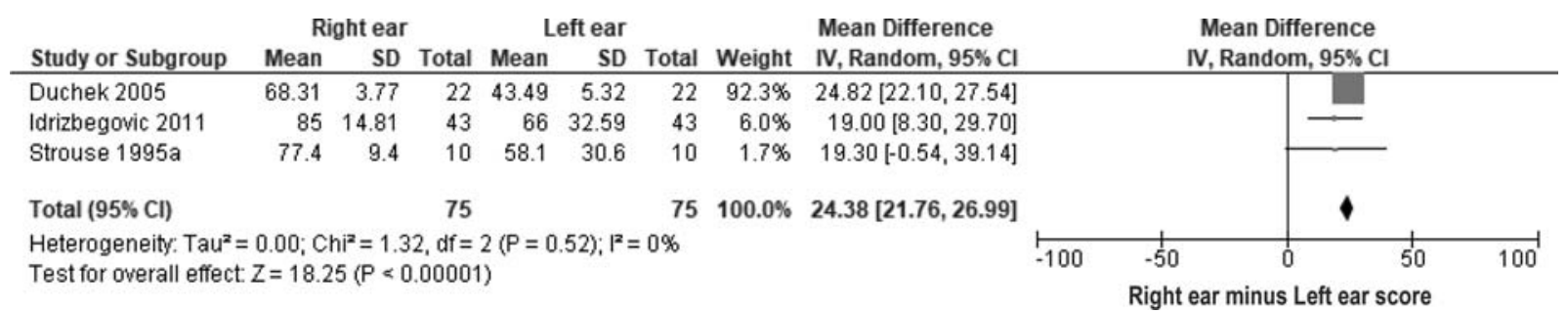

Fig. 5 The right-ear advantage score for the dementia group. For the mean difference approach, the standard deviations and the sample sizes are used together to calculate the weight given to each study. The square represents the weighted mean difference while the diamond represents the pooled mean difference. $\mathrm{Cl}$, confidence interval; IV, inverse variance; SD, standard deviation.

\section{Mild Cognitive Impairment}

Two papers were included in this quantitative analysis. ${ }^{12,31}$ The difference between the mean pooled DDT scores in each ear for the MCI group was statistically significant. The rightear mean dichotic digit score average was higher than the left ear mean score by $5.73 \%$ (95\% CI: $11.23-0.23$ ), as shown in - Fig. 6. Heterogeneity tests showed high heterogeneity among all the included studies $\left(\mathrm{X}^{2}=5.38, \mathrm{df}=1, I^{2}=81 \%\right)$.

It was not possible to perform a sensitivity analysis due to the limited numbers of the included studies. The metaanalysis of the two datasets may therefore not be appropriate due to the high heterogeneity reported above. Both papers presented a consistent difference mean dichotic digit score, with a higher score for the right ear by $3.32 \%$ (95\% CI: $4.79-$ $1.86)^{12}$ and 9.00 (95\% CI: 13.57-4.43). ${ }^{31}$

\section{Healthy Controls}

Three papers were included in this quantitative analysis. ${ }^{5,10,12}$ The difference between the mean pooled DDT scores in each ear for the healthy control group was not significant with a MD of $0.93 \%$ (95\% CI: 2.42 to -0.57), as shown in - Fig. 7. Heterogeneity tests showed low heterogeneity among all the included studies $\left(X^{2}=2.75, \mathrm{df}=2, I^{2}=27 \%\right)$.

\section{Dementia versus Mild Cognitive Impaired versus}

Noncognitively Impaired Controls

The 95\% CI and mean right-ear advantage scores (i.e., the difference in the summary mean DDT score between the right and left ears) for each population group are presented in -Fig. 8. The right-ear advantage score $95 \% \mathrm{CI}$ ranges for the dementia and control groups did not overlap, which indicates that the right-ear advantage score was significantly different between the dementia group and controls. Similarly, the 95\% CI showed a significant difference between the dementia and mild cognitively impaired groups. However, there was no significant difference in the right-ear advantage scores for the mild cognitively impaired and the noncognitively impaired controls.

\section{Discussion}

\section{Overall Summary of Evidence}

The baseline characteristics of dementia and controls were mostly comparable except for slightly lower education levels among the dementia group. This can be considered a confounding factor for the DDT analysis. However, it should be noted that the lower education level is one of the known risk

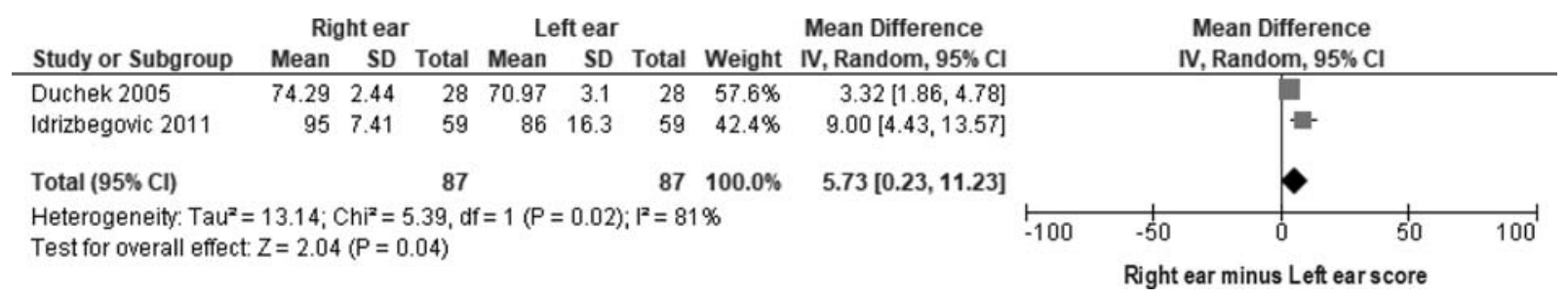

Fig. 6 The right-ear advantage score for the mild cognitive impaired group. For the mean difference approach, the standard deviations and the sample sizes are used together to calculate the weight given to each study. The square represents the weighted mean difference while the diamond represents the pooled mean difference. $\mathrm{Cl}$, confidence interval; IV, inverse variance; SD, standard deviation. 


\begin{tabular}{|c|c|c|c|c|c|c|c|c|c|c|c|c|}
\hline \multirow[b]{2}{*}{ Study or Subgroup } & \multicolumn{3}{|c|}{ Right ear } & \multicolumn{3}{|c|}{ Left ear } & \multicolumn{3}{|c|}{ Mean Difference } & \multirow{2}{*}{\multicolumn{2}{|c|}{$\begin{array}{l}\text { Mean Difference } \\
\text { IV, Random, } 95 \% \mathrm{Cl}\end{array}$}} & \\
\hline & Mean & SD & Total & Mean & SD & Total & Weight & IV, Random, $95 \% \mathrm{Cl}$ & & & & \\
\hline Duchek 2005 & 74.74 & 1.33 & 44 & 73.19 & 2.44 & 44 & $70.5 \%$ & $1.55[0.73,2.37]$ & & & & \\
\hline Idrizbegovic 2011 & 95 & 7.41 & 34 & 95 & 5.19 & 34 & $19.1 \%$ & $0.00[-3.04,3.04]$ & & & & \\
\hline Strouse 1995a & 94.4 & 5.4 & 10 & 96 & 4.5 & 10 & $10.4 \%$ & $-1.60[-5.96,2.76]$ & & & 1 & \\
\hline Total $(95 \% \mathrm{Cl})$ & & & 88 & & & 88 & $100.0 \%$ & $0.93[-0.57,2.42]$ & & & ) & \\
\hline \multicolumn{9}{|c|}{$\begin{array}{l}\text { Heterogeneity: } \mathrm{Tau}^{2}=0.65 ; \mathrm{Chi}^{2}=2.75, \mathrm{df}=2(P=0.25) ; 1^{2}=27 \% \\
\text { Test for overall effect: } Z=1.21(P=0.23)\end{array}$} & -100 & -50 & 50 & 100 \\
\hline & & & & & & & & & \multicolumn{4}{|c|}{ Right ear minus Left ear score } \\
\hline
\end{tabular}

Fig. 7 The right-ear advantage score for the noncognitively impaired controls. For the mean difference approach, the standard deviations and the sample sizes are used together to calculate the weight given to each study. The square represents the weighted mean difference while the diamond represents the pooled mean difference. $\mathrm{Cl}$, confidence interval; IV, inverse variance; SD, standard deviation.

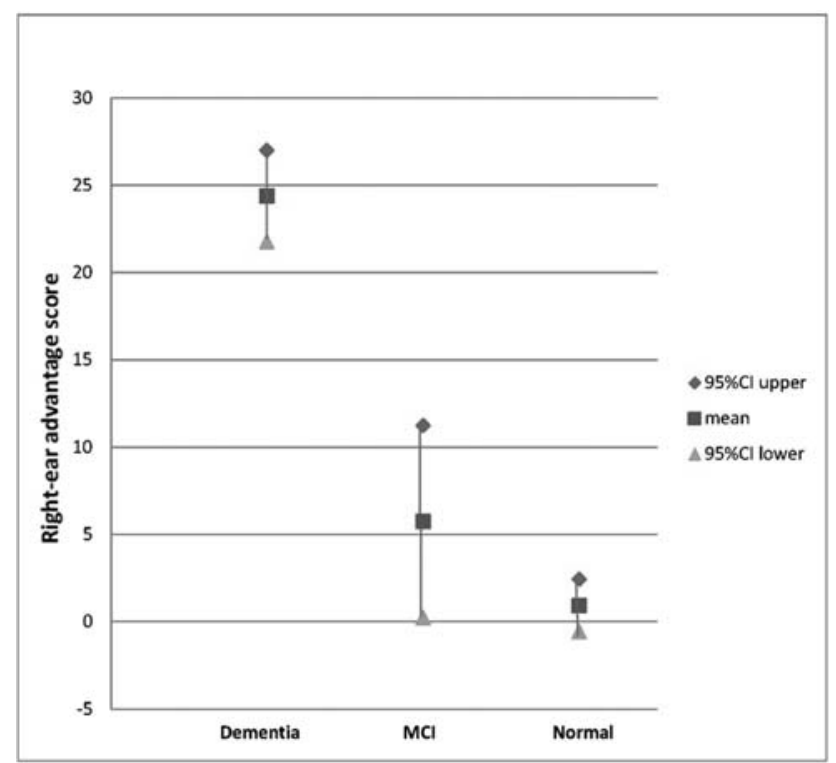

Fig. 8 Comparison of the difference between the right-ear advantage scores for the dementia, mild cognitively impaired, and noncognitively impaired groups. (Right-ear advantage scores were calculated from pooled mean right ear dichotic score minus pooled mean left ear dichotic score.)

factors for dementia. ${ }^{33}$ Overcoming this confounding factor could be challenging for future research. On the other hand, lower years of education could be related to characteristics of the dementia subjects in the cohort such as that they may have undiagnosed poor binaural integration skills throughout their lifespan, which explained their lower dichotic digit performance. Thus, causality cannot be determined in a cross-sectional study, as poorer binaural integration may contribute to later life cognitive problems.

Another possible important confounding factor for our DDT analysis is hearing loss which often accompanies dementia and aging. Therefore, to control for this factor, we performed a comparison of hearing levels between the groups within our meta-analysis of cross-sectional studies, which showed no significant difference. This may be explained by the fact that all available studies with hearing level data stated in their inclusion criteria that moderate/severe hearing loss participants along with asymmetrical hearing loss participants would be excluded. However, in the two longitudinal studies, one study reported that the hearing level at baseline and the rates of hearing decline had no differences between the two groups. ${ }^{5,31}$ The other study reported that the hearing of the cognitive impaired group was significantly worse than control at baseline but hearing test data were not reported at follow-up., ${ }^{2,9}$

All studies reported a lower dichotic digit score in patients with dementia compared with controls, and when ear advantage was measured, all studies also reported an increased right-ear advantage for patients with dementia. These effects were prominent even though several of the papers here used participants with subjective memory complaints as healthy controls. People who present with subjective memory complaints in a memory clinic, even when not meeting criteria for MCI or dementia, have a 10 times increase in the risk of dementia over 6 years compared with cognitively healthy community controls. ${ }^{34}$ A substantial proportion of these memory clinic controls may have been at a preclinical stage of $\mathrm{AD}$ or other dementia. Those adults may have also had undiagnosed binaural integration difficulties throughout development; as a result, a control group should only be composed of individuals with normal educational attainment and no evidence of memory, cognitive, or attentional factors. Therefore, the use of this population as "healthy" controls may underestimate the true effect size of DDT total scores and right-ear advantage, which may be even higher when using a truly representative cognitively healthy sample.

It was proposed by Petersen in $1999^{35}$ that the MCI population is at a precursor stage of dementia. This population can deteriorate more rapidly to the dementia stage when compared with controls. Therefore, the study of the DDT among this population may help to explore its use as a potential predictor for dementia. Despite limited data and high heterogeneity for the MCI group, we found that while the overall score was not significantly different from the control group, the right-ear advantage was significantly larger for people with $\mathrm{MCI}$ relative to controls, with increasing differences relating to increasing severity of the cognitive decline. The nonsignificant difference in overall scores between $\mathrm{MCI}$ and controls should be interpreted with caution since some of the control samples included people with subjective memory concerns as controls. Moreover, the high heterogeneity of the $\mathrm{MCI}$ group could also contribute to this nonsignificant result. This high heterogeneity was possibly due to different diagnostic criteria for this condition in each included study.

\section{Decreased Total Dichotic Digit Mean Score in People with Dementia \\ Listening to target speech in a dichotic configuration is cognitively challenging even for the healthy population.}


Therefore, it is expected that performance in this situation is even more compromised for the cognitively impaired population, putatively because there are not enough remaining cognitive resources to cope with difficult listening situations. $^{36,37}$

We have demonstrated a significantly decreased total DDT score in people with dementia compared with normal controls in our meta-analysis of cross-sectional studies. The poor ability to detect target speech (digits) in the presence of background competing speech sounds may correspond to difficulties in several everyday listening situations for the patients. These listening situations are usually categorized as similar to those seen in the classic "cocktail party" paradigm, when an individual needs to listen to an auditory target (e.g., their name) in a busy noisy party environment. This is a situation when people with AD perform worse than their age-matched peers. Functional neuroimaging research shows significant enhancement during this listening situation in the right supramarginal gyrus (inferior parietal cortex) for $\mathrm{AD}$ participants compared with healthy controls. $^{38}$ This area of the brain is suggested to be a critical locus in AD pathogenesis. ${ }^{39}$

Increased Right-Ear Advantage (Difference in Right and Left Dichotic Digit Mean Score) in Dementia

In our meta-analysis, the right-ear advantage scores were significantly higher for people with dementia than in healthy control, without any overlaps between the groups. The right ear advantage was prominent because of the decrease of the left-ear dichotic digit performance among the dementia group. This selective lower performance on the left ear may be as a result of corpus callosum changes among the dementia patients, which affects the processing of speech stimuli from the left. Corpus callosum white matter changes and/or atrophy have been proposed to associate with early neurodegenerative forms of $\mathrm{AD}$ in a neuroimaging study. ${ }^{22}$ Even though more research is needed in this area to establish this long-term temporal association, the right-ear advantage in the DDT may also index this change in AD. In our meta-analysis, participants with dementia had a dichotic digit mean score in the right ear approximately 20 percent higher than in the left ear. Participants who were not cognitively impaired did not have significantly different scores between the right and the left ear.

As for the potential use of DDT to explore a potential predementia diagnosis in the $\mathrm{MCI}$ group, both papers included here showed a consistent and significant right-ear advantage despite their high heterogeneity $\left(I^{2}=81\right)$. This right-ear advantage difference scores ranged from 4.79 to $1.86(95 \% \mathrm{CI})$ 12 and 13.57 to $4.43(95 \% \mathrm{Cl}){ }^{31}$ However, there was overlap between the right-ear advantage scores of participants with $\mathrm{MCI}$ and the noncognitively impaired population.

Participants with dementia not only had an increased right-ear advantage at baseline, but also had a further increased right-ear advantage at 1.5 years follow-up compared with controls, which was due to a left-ear dichotic digit score decrease. ${ }^{31}$ This finding of a more rapidly increased right-ear advantage over time in the AD group may suggest a higher rate of corpus callosum atrophy in patients with $\mathrm{AD} .{ }^{40}$
Our results suggest that older people with a marked rightear advantage on the DDT $>20 \%$ may require close monitoring for further signs of cognitive impairment. This is consistent with previous research that suggested that changes in DDT scores indicating a binaural integration deficit may index susceptibility for the memory and cognitive-associated problems among older adults. ${ }^{2}$ The dichotic digit score could potentially be a noninvasive test for the early detection of neurodegenerative changes, although, to our knowledge, this has not been explicitly tested yet.

\section{Possible Implication}

Overall, the DDT could represent a noninvasive, practical predictor for cognitive decline that may complement more standard cognitive testing. As it has a high repeatability even among dementia participants, ${ }^{41}$ its implementation in the dementia clinic is feasible. Further longitudinal cohort studies are needed to further investigate its potential as a screening tool for dementia.

\section{Limitations and Future Directions}

To date, there have been relatively few studies on this topic, while some studies had limited numbers of participants without prior power/sample size calculation. Further studies with more participants will facilitate more robust metaanalyses.

The majority of papers were cross-sectional studies. There was a single prospective study that showed that impairment of DDT predicted future dementia. This is a suggestive finding that requires replication in further longitudinal research.

This meta-analysis used mean and SD from each paper, which is a relatively crude approach. Using full raw datasets from each study to calculate an ear advantage score for each individual participant would yield a more precise ear advantage score and 95\% CI range for each group.

Selective decreased performance in responding to digits presented through the left ear in this population may warrant further investigation as to whether the increased rightear advantage can be a clue for future cognitive decline.

\section{Conclusions}

DDT scores for cognitively impaired patients are likely to be lower than for noncognitively impaired participants. Moreover, patients with cognitive impairment show wider rightear advantage scores compared with those of healthy participants. These findings are also more prominent when the degree of cognitive impairment increases in older adults. Further research is needed to investigate the use of the DDT ear advantage measure as an early indicator for cognitive impairment and neurodegeneration in older adults.

\footnotetext{
Author Contributions

N.U. is the main author of this paper. N.U. conducted the literature search. N.U. and D.E.B. conducted the systematic review. C.J.D.H., J.S.D., S.G.C., and J.W. contributed to the manuscript write-up. All authors read and approved the final manuscript.
} 


\section{Financial Disclosure}

No specific funding was granted for the conduct of this research.

N.U. is supported by a King Chulalongkorn Memorial Hospital (Thai Red Cross organization; Teaching hospital for Faculty of medicine, Chulalongkorn university) fellowship. C.J.D.H. is supported by an Action on Hearing LossDunhill Medical Trust Pauline Ashley fellowship. J.W. receives research funding support from the Alzheimer's Society, Alzheimer's Research UK and the NIHR UCLH Biomedical Research Centre.

\section{Conflicts of Interest}

None.

\section{References}

1 Hardy CJ, Marshall CR, Golden HL, et al. Hearing and dementia. J Neurol 2016;263(11):2339-2354

2 Gates GA, Anderson ML, McCurry SM, Feeney MP, Larson EB. Central auditory dysfunction as a harbinger of Alzheimer dementia. Arch Otolaryngol Head Neck Surg 2011;137(04): 390-395

3 Petersen RC. Mild cognitive impairment as a diagnostic entity. J Intern Med 2004;256(03):183-194

4 WHO. International Statistical Classification of Diseases and Related Health Problems, 11th Revision (ICD-11). Geneva: World Health Organization; 2019

5 Idrizbegovic E, Hederstierna C, Dahlquist M, Kämpfe Nordström C, Jelic V, Rosenhall U. Central auditory function in early Alzheimer's disease and in mild cognitive impairment. Age Ageing 2011;40 (02):249-254

6 Golob EJ, Ringman JM, Irimajiri R, et al. Cortical event-related potentials in preclinical familial Alzheimer disease. Neurology 2009;73(20):1649-1655

7 Musiek FE, Gollegly KM, Kibbe KS, Verkest-Lenz SB. Proposed screening test for central auditory disorders: follow-up on the dichotic digits test. Am J Otol 1991;12(02):109-113

8 Musiek FE. Assessment of central auditory dysfunction: the dichotic digit test revisited. Ear Hear 1983;4(02):79-83

9 Gates GA, Anderson ML, Feeney MP, McCurry SM, Larson EB. Central auditory dysfunction in older persons with memory impairment or Alzheimer dementia. Arch Otolaryngol Head Neck Surg 2008;134(07):771-777

10 Strouse AL, Hall JW III, Burger MC. Central auditory processing in Alzheimer's disease. Ear Hear 1995;16(02):230-238

11 Strouse A, Wilson RH. Stimulus length uncertainty with dichotic digit recognition. J Am Acad Audiol 1999b10(04):219-229

12 Duchek JM, Balota DA. Failure to control prepotent pathways in early stage dementia of the Alzheimer's type: evidence from dichotic listening. Neuropsychology 2005;19(05):687-695

13 Strouse A, Wilson RH. Recognition of one-, two-, and three-pair dichotic digits under free and directed recall. J Am Acad Audiol 1999a10(10):557-571

14 Kimura D. Cerebral dominance and the perception of verbal stimuli. Can J Psychol/Revue Canadienne De Psychologie 1961; 15:166-171

15 Ojemann G, Ojemann J, Lettich E, Berger M. Cortical language localization in left, dominant hemisphere. An electrical stimulation mapping investigation in 117 patients. 1989. J Neurosurg 2008;108(02):411-421

16 Zenker F, Espino R, Cosials S, Carmona G, Belda R, De Prat JJ. The effect of age over the dichotic digit test. Paper presented at: 8th EFAS Congress/10th Congress of the German Society of Audiology, Heidelberg, Germany, June 6-9, 2007
17 Aiello I, Sotgiu S, Sau GF, Manca S, Conti M, Rosati G. Long latency evoked potentials in a case of corpus callosum agenesia. Ital J Neurol Sci 1994;15(09):497-505

18 Landry SP, Fuente A. Dichotic listening deficit associated with solvent exposure. Otol Neurotol 2017;38(06):e68-e74

19 Adibpour P, Dubois J, Moutard ML, Dehaene-Lambertz G. Early asymmetric inter-hemispheric transfer in the auditory network: insights from infants with corpus callosum agenesis. Brain Struct Funct 2018;223(06):2893-2905

20 Hannay HJ, Walker A, Dennis M, Kramer L, Blaser S, Fletcher JM. Auditory interhemispheric transfer in relation to patterns of partial agenesis and hypoplasia of the corpus callosum in spina bifida meningomyelocele. J Int Neuropsychol Soc 2008;14(05): 771-781

21 Staffaroni AM, Elahi FM, McDermott D, et al. Neuroimaging in dementia. Semin Neurol 2017;37(05):510-537

22 Hampel H, Teipel SJ, Alexander GE, et al. Corpus callosum atrophy is a possible indicator of region- and cell type-specific neuronal degeneration in Alzheimer disease: a magnetic resonance imaging analysis. Arch Neurol 1998;55(02):193-198

23 Bartzokis G, Lu PH, Geschwind DH, Edwards N, Mintz J, Cummings $\mathrm{JL}$. Apolipoprotein $\mathrm{E}$ genotype and age-related myelin breakdown in healthy individuals: implications for cognitive decline and dementia. Arch Gen Psychiatry 2006;63(01):63-72

24 Tuwaig M, Savard M, Jutras B, et al; PREVENT-AD Research Group. Deficit in central auditory processing as a biomarker of pre-clinical Alzheimer's disease. J Alzheimers Dis 2017;60(04):1589-1600

25 NIH, N. I. O. H., National Heart Lung and Blood Institute. 2014NIH quality assessment tool for observational cohort and cross-sectional studies. Study quality assessment tools Available at: https://www.nhlbi.nih.gov/health-topics/study-quality-assessment-tools. Accessed August 25, 2020

26 Biljana M, Jelena M, Branislav J, Milorad R. Bias in meta-analysis and funnel plot asymmetry. Stud Health Technol Inform 1999; 68:323-328

27 Higgins J, Green S, eds. Cochrane Handbook for Systematic Reviews of Interventions Version 5.1.0 [updated March 2011] London: The Cochrane Collaboration; 2011

28 REVIEWMANAGER. Review Manager. 5.3 ed. Copenhagen: The Nordic Cochrane Centre, The Cochrane Collaboration; 2014

29 DerSimonian R, Laird N. Meta-analysis in clinical trials. Control Clin Trials 1986;7(03):177-188

30 Bouma A, Gootjes L. Effects of attention on dichotic listening in elderly and patients with dementia of the Alzheimer type. Brain Cogn 2011;76(02):286-293

31 Idrizbegovic E, Hederstierna C, Dahlquist M, Rosenhall U. Shortterm longitudinal study of central auditory function in Alzheimer's disease and mild cognitive impairment. Dement Geriatr Cogn Disord Extra 2013;3(01):468-471

32 Gates GA, Gibbons LE, McCurry SM, Crane PK, Feeney MP, Larson EB. Executive dysfunction and presbycusis in older persons with and without memory loss and dementia. Cogn Behav Neurol 2010;23(04):218-223

33 Livingston G, Sommerlad A, Orgeta V, et al. Dementia prevention, intervention, and care. Lancet 2017;390(10113):2673-2734

34 Slot RER, Sikkes SAM, Berkhof J, et al; Alzheimer's Disease Neuroimaging Initiative DESCRIPA working group INSIGHT-preAD study group SCD-I working group. Subjective cognitive decline and rates of incident Alzheimer's disease and non-Alzheimer's disease dementia. Alzheimers Dement 2019;15(03):465-476

35 Petersen RC, Smith GE, Waring SC, Ivnik RJ, Tangalos EG, Kokmen E. Mild cognitive impairment: clinical characterization and outcome. Arch Neurol 1999;56(03):303-308

36 CHABA. Speech understanding and aging. Working Group on Speech Understanding and Aging. Committee on Hearing, Bioacoustics, and Biomechanics, Commission on Behavioral and Social Sciences and Education, National Research Council. J Acoust Soc Am 1988;83(03):859-895 
37 Lindenberger $U$, Baltes PB. Sensory functioning and intelligence in old age: a strong connection. Psychol Aging 1994;9(03):339-355

38 Golden HL, Agustus JL, Goll JC, et al. Functional neuroanatomy of auditory scene analysis in Alzheimer's disease. Neuroimage Clin 2015;7:699-708

39 Warren JD, Fletcher PD, Golden HL. The paradox of syndromic diversity in Alzheimer disease. Nat Rev Neurol 2012;8(08):451-464
40 Elahi S, Bachman AH, Lee SH, Sidtis JJ, Ardekani BAAlzheimer's Disease Neuroimaging Initiative. Corpus callosum atrophy rate in mild cognitive impairment and prodromal Alzheimer's disease. J Alzheimers Dis 2015;45(03):921-931

41 Strouse AL, Hall JW III. Test-retest reliability of a dichotic digits test for assessing central auditory function in Alzheimer's disease. Audiology 1995;34(02):85-90 